\title{
Phase dynamics for the wavy vortex state of the Taylor instability
}

\author{
H. Brand and M. C. Cross \\ Bell Laboratories, Murray Hill, New Jersey 07974
}

(Received 22 October 1982)

\begin{abstract}
We present dynamic equations for the slow macroscopic variables of the wavy vortex state. The static solutions explain in a qualitative way recent experimental results of Ahlers et al on the variation of the vortex diameter throughout the cell. In addition, we predict the existence of a pair of propagating or overdamped normal modes (depending on the wave vector of the disturbance) formed by the slow variables.
\end{abstract}

The Couette-Taylor system, consisting of a fluid contained between two concentric cylinders with the inner one rotating, is particularly convenient for the experimental investigation of the sequence of hydrodynamic instabilities leading to turbulent behavior (for a recent review see Ref. 1). Above a critical rotation rate the uniform (Couette) flow is unstable to the Taylor state of azimuthal vortices. The first time-dependent state, in which periodic displacements in the vortices propagate around the cylinders, tends to occur for only slightly larger rotation rates. In this paper we study the static and dynamic behavior of slow perturbations of this "wavy vortex flow," with the Taylor vortex flow a special case. This work was motivated to a large degree by the recent observation by Ahlers et al. ${ }^{2}$ that the wavelength of the vortices in the wavy state is not constant over the cell, but varies over a rather long length scale (of order ten rolls). This is in sharp contrast to the situation in Rayleigh-Benard convection, where the roll wavelength in similar configurations is essentially constant over the bulk of the cell. In addition to accounting for this observation, we predict a new mode for variations of the vortex diameter that is propagating at long wavelengths, and becomes overdamped, and finally diffusive as the wavelength of the perturbation is decreased. In the course of this work we explore the analogies with the hydrodynamic description of slow perturbations in equilibrium systems. We also present for the first time the full two-dimensional amplitude equation describing the behavior of the Taylor vortex state near onset in the small gap limit.

Our method of approach is to construct using general symmetry arguments coupled equations for the slowly varying "phase" variables characterizing the wavy state. Two phases are necessary: the first $\psi$ giving the position of the vortices along the axis; the second $\Phi$ giving the azimuthal position of the waves. These phases would be identified as the variables characterizing the spontaneously broken continuous symmetries in an equilibrium system. This description of the wavy flow should be contrasted with the "phase diffusion" equation for the single slow phase variable in Rayleigh-Benard convection. ${ }^{3-5}$ The various coefficients in the equations are estimated in the limit of a small gap between the cylinders using the proximity of the wavy state to the onset of the Taylor vortex state. This generalizes the procedure of Davey et $a l .{ }^{6}$ to a spatially nonuniform wavy flow state.

The main result of the present Communication is the set of dynamic equations for the two phases $\Phi$ and $\psi$ :

$$
\begin{aligned}
& \dot{\psi}=D_{1} \psi^{\prime \prime}+C_{1}\left(q_{y}\right) \Phi^{\prime}, \\
& \dot{\Phi}=D_{2} \Phi^{\prime \prime}+C_{2}\left(q_{y}\right) \psi^{\prime},
\end{aligned}
$$

where the dot denotes a time derivative and the prime a spatial derivative along the axis ( $x$ direction). These equations are given by the restriction that they reduce to a simple phase diffusion equation in the Taylor vortex state, and invariance under the symmetry $x \rightarrow-x, \psi \rightarrow-\psi$. In Eqs. (1) and (2) we have ignored azimuthal ( $y$ direction) inhomogeneities in the phases. The parameter $q_{y}$ is the azimuthal wave number of the wavy vortex state: Although all parameters depend on $q_{y}$, in general, we have emphasized the $q_{y}$ dependence of the cross-coupling coefficients $C_{1}, C_{2}$, since the symmetry $y \rightarrow-y$, $q_{y} \rightarrow-q_{y}, \psi \rightarrow \psi, \Phi \rightarrow-\Phi$, and the assumption of analyticity imply $C_{1}, C_{2} \propto q_{y}$ for small $q_{y}$. Note that $\psi^{\prime}$ gives the change in the vortex wave number, and $\dot{\Phi}$ the change in the frequency of the wavy motion.

To study the static solutions of Eqs. (1) and (2) it is useful to add a phenomenological source term $(\delta \Omega) \delta(x)$ to Eq. (2) corresponding to a local tendency to increase the wavy mode frequency by $\delta \Omega$ at $x=0$. The solutions are then

$$
\begin{aligned}
& \delta k=k-k_{\infty}=\delta k_{0} \exp (-|x| / l), \\
& \delta \Phi=\Phi-\Phi_{\infty}=\left(D_{1} / C_{1}\right) \delta k
\end{aligned}
$$

with $k$ the local wave vector and $k_{\infty}$ the value at large distances from the source, and $\delta k_{0}$ proportional to the source strength $\delta \Omega$. The "penetration length" $l$ 
is given by

$$
l^{2}=D_{1} D_{2} / C_{1} C_{2}
$$

and becomes long (justifying the use of the phase equations) for $C_{1}, C_{2}$ small.

Equations (1) and (2) may now be applied to the observations of Ahlers et al. Here a local tendency towards a different wave propagation speed due to end perturbations may be expected to drive a distortion in $\Phi(x)$ and hence, through Eq. (1), a local perturbation of the wave vector. Although the details of the resulting source terms in Eqs. (1) and (2) are not clear, the value of the penetration length $l$, and the relationship between $\delta \Phi$ and $\delta k$ are independent of them; and Eqs. (3) and (4) should apply near each end. The long penetration length for the wave number perturbation observed by Ahlers et al. may therefore be ascribed directly to the coupling to the azimuthal phase.

The time evolution of slow disturbances of the wave number can also be studied from the phase equations. Introducing a perturbation proportional to $\exp [i(K x-\omega t)]$ we find a propagating mode for $K \rightarrow 0$ with frequency $\omega= \pm \sqrt{C_{1} C_{2}} K$ and $0\left(K^{2}\right)$ damping. For larger $K$ we get

$\omega=-\frac{1}{2} i\left(D_{1}+D_{2}\right) K^{2} \pm \frac{1}{2} K\left[4 C_{1} C_{2}-K^{2}\left(D_{1}-D_{2}\right)^{2}\right]^{1 / 2}$

for $4 C_{1} C_{2}>K^{2}\left(D_{1}-D_{2}\right)^{2}$, and

$\omega=-i\left\{\frac{1}{2}\left(D_{1}+D_{2}\right) \pm \frac{1}{2} K\left[K^{2}\left(D_{1}-D_{2}\right)^{2}-4 C_{1} C_{2}\right]^{1 / 2}\right\}$

for $4 C_{1} C_{2}<K^{2}\left(D_{1}-D_{2}\right)^{2}$. Thus we find by increasing the wave vector, first, a propagating mode, then an overdamped mode with a velocity dependent on the damping coefficients, and, finally, a pure diffusive mode. ${ }^{7}$ Note that we assume $C_{1} C_{2}$ to be positive: This result follows from the analysis presented later in the small gap limit near the onset of the wavy mode and is required for stability. $C_{1}$ or $C_{2}$ passing through zero well above threshold would indicate the onset of an additional instability. It is interesting to point out that for a system near thermodynamic equilibrium the structure of Eqs. (1) and (2), including relationships between the coefficients, would be pinned down by the behavior of $\psi, \Phi$ under spatial inversion and time reversal. ${ }^{8}$ For the present nonequilibrium system the situation is slightly different. To derive Eqs. (1) and (2) we have only used the symmetry $x \rightarrow-x$ and $\psi \rightarrow-\psi, \Phi \rightarrow \Phi$. It is a posteriori that we can say, taking into account the timereversal symmetry of $\psi$ and $\Phi$, that $C_{1}$ and $C_{2}$ are reversible, whereas $D_{1}$ and $D_{2}$ are dissipative. This transformation has not, however, entered our derivation of the basic equations in any way.

The coefficients in Eqs. (1) and (2) may be rough- ly estimated by considering the small gap limit, using the following two observations:

(i) Although the first instability of Couette flow is to the stationary Taylor vortex state, at slightly higher Taylor numbers Couette flow is also unstable to vortices with azimuthally propagating waves. The wavy vortex state may be understood by considering the rather delicate competition between the $q_{y}=0$ state and a state with nonzero $q_{y}{ }^{6}$

(ii) Since the wavy vortex state first occurs close to the first instability in the small gap limit, its onset may be approximately treated using a lowest order "amplitude equation" found by expanding about the critical Taylor number $T_{c}$ for the Taylor vortex state $\left(T_{c}=3390\right)$.

The amplitude equation may be derived using the general procedure of Newell. ${ }^{9}$ This leads to the equation at lowest order in $\epsilon=\left(T-T_{c}\right) / T_{c}$ :

$$
\begin{aligned}
\partial_{\tau} A= & \tau_{0}^{-1}\left[A+\left(\xi_{x}^{2} \partial_{X}^{2}+\xi_{y}^{2} \partial_{Y}^{2}\right) A-g|A|^{2} A\right] \\
& +i \sqrt{T_{c}} s_{1} \partial_{X} \partial_{Y} A,
\end{aligned}
$$

where $A(X, Y, \tau)$ is the complex amplitude function in terms of which the deviations of the fluid velocities from Couette flow take the form (e.g., for the radial velocity $w$ )

$$
w=\epsilon^{1 / 2}\left[A e^{i k_{0} x}+\text { c.c. }\right] w_{0}(z)+0(\epsilon),
$$

with $w_{0}(z)$ a known function. The variables $X$ $=\epsilon^{1 / 2} x, Y=\epsilon^{1 / 2}\left(y-s_{0} \sqrt{T_{c}} \tau\right)$ with $y=\left(\frac{1}{2} \delta\right)^{-1 / 2} \theta$ and $\tau=\epsilon t$ are slow length and time scales with $x$ the axial coordinate scaled by the gap $d, \theta$ the azimuthal angle, and $\tau$ the time scaled with $d^{2} / \nu$, with $\nu$ the viscous diffusivity. The parameter $\delta$ is the ratio of the gap to the average radius of the cylinders. In the small gap limit $\delta \rightarrow 0$ the azimuthal variation becomes "slow" in the $y$ coordinate, even though periodic in $\theta$. $Y$ is the (scaled) azimuthal coordinate moving with the waves. The angular propagation speed of the waves at onset, expressed as a fraction of the rotation rate $\Omega$, is $s_{0}$, and the coefficient $s_{1}$ gives the change in this speed when the axial wave number is changed. In deriving Eq. (8) we have made use of the explicit form of the linear operator in the small gap limit. ${ }^{6}$ The amplitude equation [Eq. (8)] takes the familiar form except for the additional last term: It is this term that leads to the phase coupling we seek.

The wavy vortex state is given ${ }^{6}$ by the superposition

$$
A=|B| e^{i \psi}+i|C| e^{i \psi}\left(e^{i(Q Y+\Phi)}+\text { c.c. }\right) \text {, }
$$

where $|C| \rightarrow 0$ at $\epsilon_{w}$, the value of $\epsilon$ at the onset of the wavy state, and we neglect harmonics in this limit. This equation precisely defines the phases $\psi, \Phi$ introduced earlier. Equations (1) and (2) for the slow dynamics about the wavy vortex state are found after eliminating the fast variables $|B|,|C|$, with the identities $D_{1}=D_{2}=\tau_{0}^{-1} \xi_{x}^{2}, C_{2}(q)=\sqrt{T_{c}} s_{1} q$, and 
$C_{1}(q)=C_{2}(q) r^{2}$, with $r^{2}=|C|^{2} /\left(|C|^{2}+\frac{1}{2}|B|^{2}\right)$.

From the calculations of Davey et al. ${ }^{6}$ we find

$$
|C|^{2} /|B|^{2}=\xi_{y}^{2} q_{y}^{2}\left(\epsilon-\epsilon_{w}\right) / \epsilon \epsilon_{w} .
$$

Note that $C_{1}(q)$ goes to zero at $\epsilon=\epsilon_{w}$ as would be expected, but that the other parameters are independent of $\epsilon$ in the limit we consider (i.e., $\epsilon-\epsilon_{w}$ small).

We estimate the values of the parameters in Eq. (8) $\left(\tau_{0}^{-1} \sim 13, \xi_{x}^{2} \simeq 0.15, \xi_{y}^{2} \simeq 0.14\right.$, and $\left.s_{0} \simeq 0.53\right)$ from Chandrasekhar, ${ }^{10}$ and the tabulated results of Davey et al. ${ }^{6}$ and Krueger et al. ${ }^{11}$ The important coefficient $s_{1}$ is not given by these authors: Instead we estimate $s_{1} \simeq 0.014$ directly from measurements by Ahlers et al. ${ }^{1}$ at $T / T_{c}=1.44$ for the $m=3$ state with $\delta=0.1$. These values lead to the prediction for the healing length

$$
l=2.4 q_{y}^{-1} r^{-1},
$$

which diverges for small $q_{y}$ as well as for small modulation ratio $r=|C| /|B|$.

Substituting the values appropriate to the experiments of Ahlers et al. into these equations we estimate the value $l \sim 6$. From the experimental data we estimate $l \sim 10$ (although there is considerable asymmetry between the top and bottom of the cell that is not understood). This discrepancy may be due to the approximations made in evaluating the parameters, namely, the small gap limit and the proximity to onset of the wavy flow, which may well not apply for these experiments. For a better test of the quantitative predictions of the amplitude equation experiments nearer the onset of the wavy vortex state would be highly desirable.

To summarize, we have discussed the phase dynamics of the Taylor wavy mode and have found a unique structure for its dynamics, which has not been found for any hydrodynamic instability before. This unique structure is mainly due to the fact that one has two scalar quantities characterizing the longwavelength, low-frequency behavior of this system, one even, one odd, under spatial inversion along the cylinder axis. This situation is to be contrasted, for example, with the onset of convection or with the single model laser where one has only one phase variable giving rise to a purely dissipative slow dynamics. The predictions concerning the propagating and overdamped character of the normal modes in the wavy mode are certainly a new challenge for future experiments.

One of us (H.B.) acknowledges the support of the Deutsche Forschungsgemeinshaft.
${ }^{1}$ R. C. diPrima and H. L. Swinney, in Hydrodynamic Instabilities and the Transition to Turbulence, Topics in Applied Physics, edited by H. L. Swinney and J. P. Gollub (Springer, New York, 1981), Vol. 45.

${ }^{2}$ G. Ahlers, D. S. Cannell, and M. A. Dominguez Lerma, Phys. Rev. Lett. 48, 368 (1982); and (unpublished).

${ }^{3}$ Y. Pomeau and P. Manneville, J. Phys. (Paris) Lett. 40, 609 (1979)

${ }^{4}$ J. E. Wesfreid and V. Croquette, Phys. Rev. Lett. 45, 634 (1980).

${ }^{5}$ A. C. Newell and J. C. Whitehead, J. Fluid Mech. 38, 279 (1969).

${ }^{6}$ A. Davey, R. C. diPrima, and J. T. Stuart, J. Fluid Mech. 31, 17 (1968).
${ }^{7}$ We mention in passing that Eqs. (6) and (7) are similar in structure to the results obtained for the orbit waves in ${ }^{3} \mathrm{He}-A$. [See H. Brand, M. Dorfle, and R. Graham, Ann. Phys. (N.Y.) 119, 434 (1979).]

${ }^{8} \mathrm{D}$. Forster, Hydrodynamic Fluctuations, Broken Symmetry, and Correlation Functions (Benjamin, Massachusetts, 1975).

${ }^{9}$ A. C. Newell, in Lectures in Applied Mathematics, edited by M. Kac (American Mathematical Society, Providence, R.I., 1974), Vol. 15, p. 157.

${ }^{10} \mathrm{~S}$. Chandrasekhar, Hydrodynamic and Hydromagnetic Stability (Clarendon, Oxford, 1961).

${ }^{11}$ E. R. Krueger, A. Gross, and R. C. diPrima, J. Fluid Mech. 24, 52 (1966). 